\title{
Psychological impact of anti-VEGF treatments for wet macular degeneration-a review
}

\author{
Hugo Senra ${ }^{1}$ - Zaria $\mathrm{Ali}^{2} \cdot$ Konstantinos Balaskas $^{2} \cdot$ Tariq Aslam ${ }^{1,2,3}$
}

Received: 21 October 2015 /Revised: 5 April 2016 / Accepted: 10 May 2016 /Published online: 4 June 2016

(C) The Author(s) 2016. This article is published with open access at Springerlink.com

\begin{abstract}
Purpose To review the current literature on the psychological impact of anti-VEGF treatments for wet age-related macular degeneration (wAMD), in terms of patients' experiences of receiving these treatments, and the impact of these treatments for patients' mental health and quality of life.

Methods We critically analyzed current literature evaluating psychological impact of anti-VEGF treatments for wAMD. Primary searches of PubMed, Science Direct, and Web of Science were conducted in July and August of 2015. We reviewed all papers on the topic published until August 5, 2015.

Results Our literature search found 14 papers addressing the psychological impact of anti-VEGF treatments for wAMD. Results highlighted potential anxieties and experiences of pain caused by receiving regular intravitreal injections. A positive visual outcome of anti-VEGF therapy is associated with positive vision-related QOL outcomes, although such association seems to be dependent on improvements on visual acuity. In the literature reviewed, patients receiving anti-VEGF treatments showed a prevalence rate of depression between 20 and $26 \%$. Conclusions Although anti-VEGF treatments can cause some anxiety and being experienced as a stressful event, especially in the beginning of the treatment, preliminary findings suggest a potential benefit for long-term vision-related quality of life. Further longitudinal and qualitative research should bring
\end{abstract}

Hugo Senra

hugo_senra@hotmail.com

1 Institute of Human Development, University of Manchester, Oxford Road, Manchester M13 9PL, UK

2 Manchester Royal Eye Hospital, Manchester, UK

3 Heriot Watt University, Edinburgh, UK more evidence on the positive and negative effects of these treatments on patients' long-term mental health.

Keywords Wet age-related macular degeneration .

Anti-VEGF treatment · Psychological impact · Intra-vitreal injections

\section{Introduction}

Age-related macular degeneration (AMD) is currently the leading cause of irreversible vision loss and blindness in people aged 50 and older, particularly in the developed world [1]. AMD can be a highly disabling condition, causing impairment to the activities of daily living, invoking emotional distress, anxiety, and depression [2]. Currently, wet macular degeneration (wAMD) is the only form of AMD that is treatable, usually involving the use of vascular endothelial growth factor inhibitors (anti-VEGF) such as ranibizumab, aflibercept, or bevacizumab [3]. These treatments are regarded as having great potential for halting disease progression and for reducing further risk of blindness [3].

However, these treatments are administrated by invasive intra-vitreal injections, often at the conclusion of lengthy, frequent, and oft-repeated visits after transport to an eye clinic. The specific act of an intraocular anti-VEGF injection can be experienced by patients as a stressful event, especially in the beginning of treatment $[4,5]$. Furthermore, anti-VEGF treatments are frequently without a pre-established date for their completion, and this concept can entail additional anxiety to some patients.

In order to optimize patients' quality of life, it would seem prudent to not only target the impact of treatments on vision but also the impact of the repeated injection visits themselves on patients' well-being and mental health. It is therefore 
crucial to understand how patients experience these invasive treatments and how they manage any related anxieties. Additionally, it is relevant to investigate the separate issue of whether the protective effect that these treatments have on vision is also followed by any benefits on patients' mental health and quality of life, or if the treatment benefits are exclusive to patients' vision. Both questions are of importance for everyday clinical management of these patients, but unfortunately both are still poorly answered due to paucity of relevant research.

\section{Methods}

With this review, we want to critically analyze the current literature on the psychological impact of anti-VEGF treatments for wAMD. We selected all studies that fulfilled the following inclusion criteria: articles published in English; peer-reviewed articles; studies of adults with wAMD; studies addressing anti-VEGF treatments for wAMD; studies focused on the psychological and psychosocial implications of receiving anti-VEGF treatments for wAMD including the experience of receiving treatment, quality of life, anxiety, stress, and depression. We only considered studies investigating psychological consequences of receiving anti-VEGF injections for wAMD. Studies addressing the psychological impact of wAMD without controlling for intravitreal injections or studies conducted with patients who did not receive these treatments were excluded. We also excluded studies exclusively focused on pain levels induced by anti-VEGF injections or on factors associated with pain caused by injections because we do not consider pain as a psychological variable and therefore it cannot be regarded as part of the psychological impact of anti-VEGF treatment. Systematic reviews were considered in this review as they summarize pre-existent literature and evidence on the topic.

Two authors (H.S. and Z.C.A.) systematically conducted a search of electronic databases (PubMed, Web of Science, and Science Direct) to retrieve all articles published up to August 5,2015 . We searched these databases using terms that are often used in literature to designate AMD, anti-VEGF treatments and its psychological impact, including "macular degeneration" and "anti-VEGF"; "macular degeneration" and "psychological"; "macular degeneration" and "depression"; "macular degeneration" and anxiety"; "antiVEGF" and "experience"; "anti-VEGF" and "psychological"; "anti-VEGF and "depression"; "antiVEGF" and "anxiety"; "anti-VEGF" and "quality of life"; "intravitreal" and "anxiety"; "intravitreal" and "depression"; "intravitreal" and "psychological"; "intravitreal" and "quality of life". Additional sources were identified through cited and citing articles.
Two authors (H.S. and Z.C.A.) independently reviewed titles and abstracts and then the full-text articles to identify the eligible studies. Results of both researchers were compared, and clearly non-eligible studies were excluded. Then, duplicates were removed from the list. Next, the same researchers read the abstracts of the remaining articles to determine whether they met inclusion criteria. Abstracts providing sufficient detail for exclusion were removed, and the remaining full-text articles were retrieved. Full-text articles were read to determine inclusion, and disagreements were resolved via consensus and returning to the articles. Data were analyzed and summarized using a specific table (Table 1).

\section{Results}

Figure 1 describes the process of study selection. We identified 282 articles from the databases, of which 195 were excluded on the basis of title review, leaving 87 articles. Of 87 articles, we excluded 35 for being duplicates and 40 for not meeting our eligibility criteria, with 12 articles remaining for review. Reference lists were reviewed using the same process, and two additional articles were identified for inclusion, leaving a final list of 14 articles to be reviewed [4-17]. The main reasons for not meeting the eligibility criteria of this review were samples not exclusively composed of patients with wAMD, studies about the psychological impact of antiVEGF in other medical conditions, or studies exclusively focused on other consequences of wAMD such as pain or on the effect of anesthetics on pain levels experienced by patients.

Table 1 summarizes all articles included in the current review. The year of publication ranges from 2009 to 2015 . Six articles reported observational non-interventional studies $[10-14,17]$ and three articles reported qualitative studies [5, $7,9]$. The remaining articles reviewed were one systematic review [4], one prospective clinical survey [6], one prospective cases series case series study [8], one cross-sectional study [15], and one longitudinal study [16]. With regards to the type of anti-VEGF treatment received by patients, ranibizumab was used in eight studies [6, 8-13, 15], while bevacizumab was only used in two studies [7, 17]. One study used ranibizumab and bevacizumab [16] and two other studies did not state which type of anti-VEGF had been administrated to patients $[5,14]$. The systematic review reported studies in which ranibizumab and bevacizumab were used [4]. Studies included in this review investigated the psychological impact of anti-VEGF treatments by exploring one of the following topics: (1) Patients' experiences or perspectives of the process of receiving anti-VEGF treatments for wAMD [4-9]; (2) Longer-term impact of anti-VEGF treatments on patients' quality of life [10-13]; and (3) Depression/anxiety after receiving anti-VEGF treatments for wAMD [14-17]. 


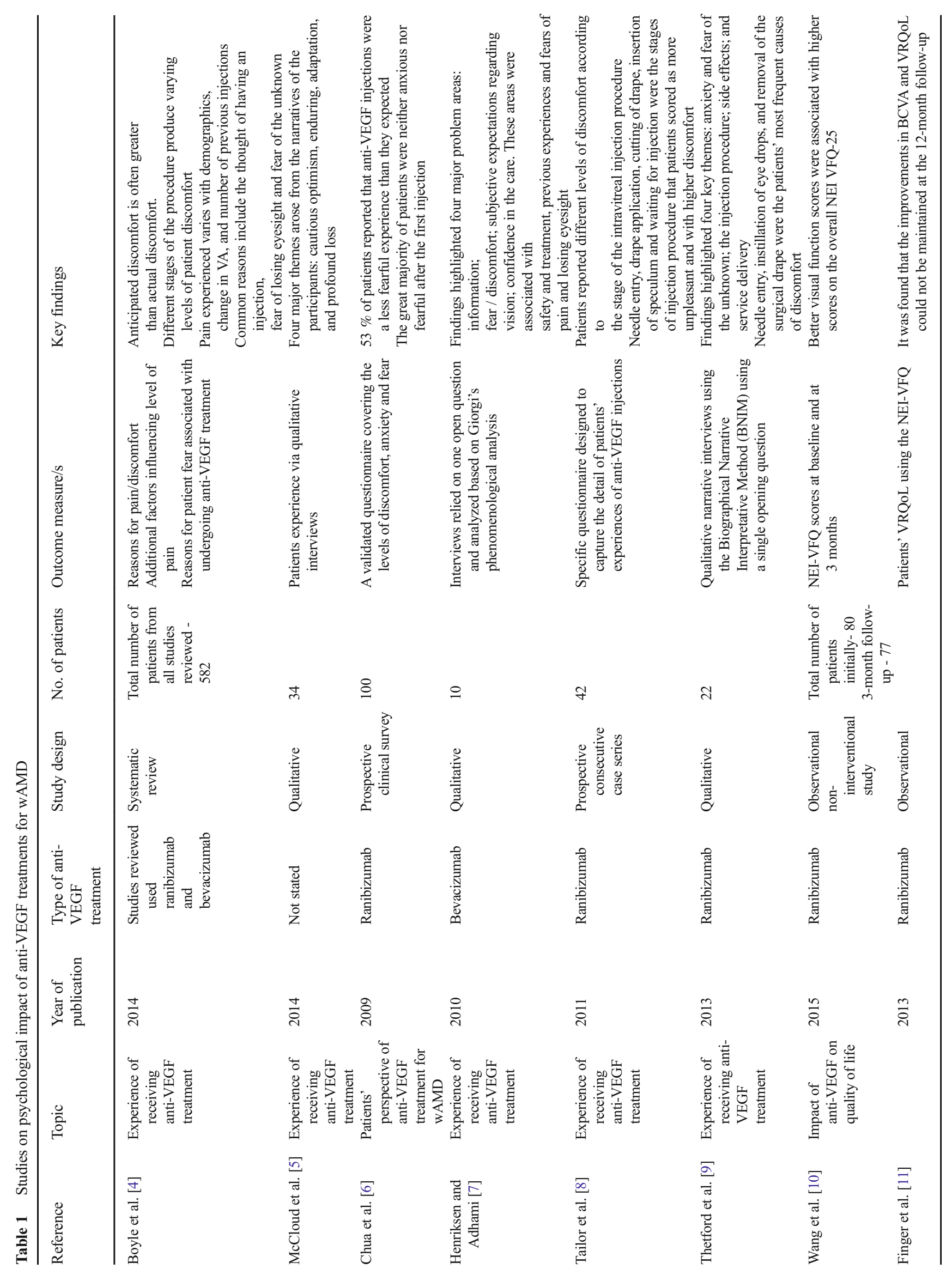




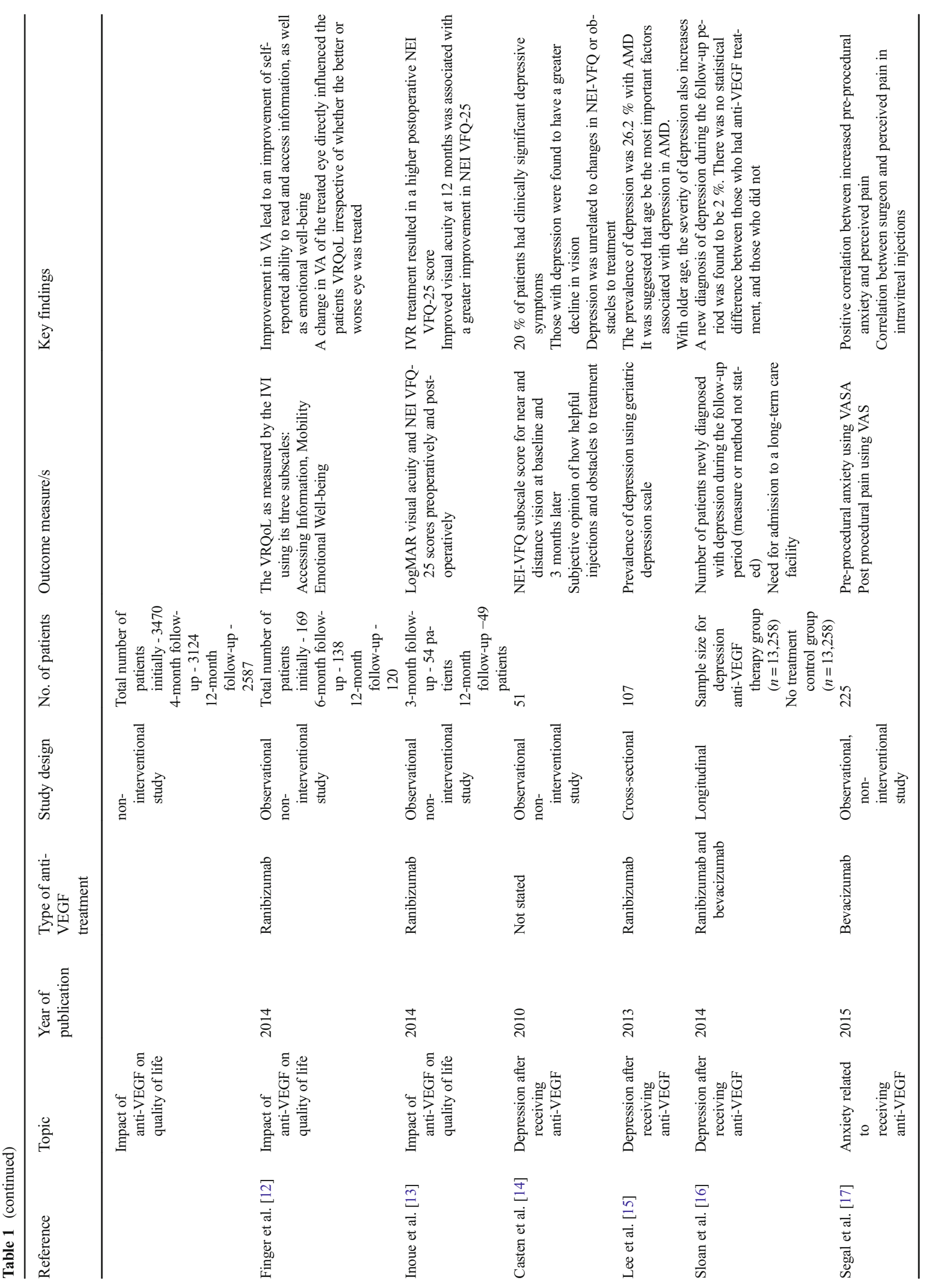


Fig. 1 Flow diagram of articles selection for review

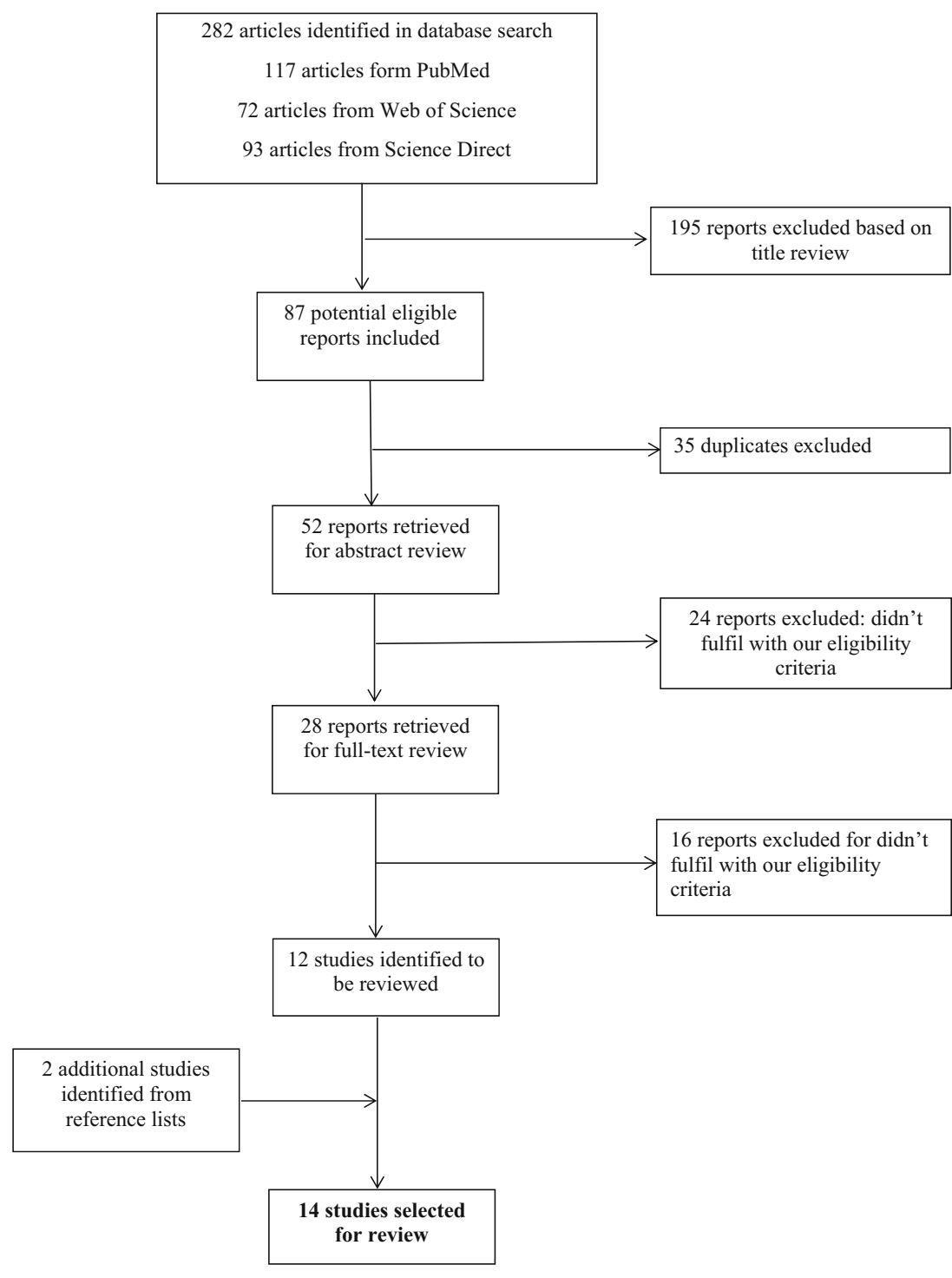

\section{Patients' experiences of the process of receiving anti-VEGF treatments for wAMD}

Our review found six articles on patients' experiences or perspectives of receiving anti-VEGF treatments for wAMD [4-9]. Findings generally highlighted frequent experiences of discomfort and fear associated with receiving anti-VEGF treatment over time [4, 5, 7-9]. The aspects of the treatment in which patients reported more discomfort were: needle entry; application of drops; insertion of speculum; waiting for injection; experiences of pain, fear of losing sight, fear for the unknown, and side effects. The most recent study we found on patient experiences of anti-VEGF highlighted the complexity and diversity of patients' experiences of treatment. This study suggested, for the first time, patients" "cautious optimism" as a valid response to treatment success. However, one study found that discomfort and fearful experiences were mainly circumscribed to the first injection and strongly related to the patients' previous expectations of treatment [6]. Finally, the systematic review on the experiences of anti-VEGF treatments [4] included four studies that were also analyzed by us [6-9] and six other studies focused on pain levels caused by anti-VEGF treatment and the effect of anesthetics on pain experienced by patients [8, 18-22]. Findings highlighted the fact that patients' expectations of pain before they started the treatment were higher than the pain experienced when receiving the treatment. Finally, the review suggested the need for more research, particularly to clarify the reasons underlying patients' anxieties when receiving anti-VEGF treatments, and more qualitative studies addressing patients' experiences of receiving the treatments.

\section{Longer-term impact of anti-VEGF treatments on patients' quality of life}

Studies reviewed found an improvement on patients' visionrelated quality of life after they have started receiving 
intravitreal treatment with ranibizumab for wAMD [10-13]. This relationship was found to be very dependent on improvements in patients' visual acuity [10-13]. One study found an improvement on patients' VRQL at month 3 of treatment [10] and another study found improvements at month 4 of treatment [11]. Two other studies found better vision-related quality of life after 12 months of treatment [11, 13]. Of four articles identified, three used the National Eye Institute Visual Function Questionnaire (NEI VFQ-25) [23] to assess patients' vision-related quality of life. This instrument comprises the assessment of general health, quality of vision, and visionrelated quality of life that includes dependency, role limitations, mental health, social functioning, ocular pain, and driving. In general these studies found an improvement on patients' vision-related quality of life after they have started receiving intravitreal treatment with ranibizumab for wAMD. An improvement on patients' mental health was also associated with receiving these treatments [10-13].

\section{Depression and anxiety after anti-VEGF treatments for WAMD}

A preliminary study has explored depression in wAMD patients receiving anti-VEGF treatments [14]. In this study, depression was assessed using the NEI VFQ [23]. Results suggested a slightly lower prevalence rate of depression among patients receiving anti-VEGF treatments in comparison with previous studies in which depression rates were not adjusted for anti-VEGF treatments. Additionally, patients with depression were found to have a greater decline in vision. Finally, depression was unrelated to changes in NEI-VFQ or obstacles to treatment.

With different results, a study conducted with 107 Korean patients with wAMD receiving intravitreal ranibizumab treatment found a prevalence of depression of $26.2 \%$, which is consistent with the literature pre-anti-VEGF treatments [15]. In this study, depression was assessed using the Geriatric Depression Scale [24].

A retrospective study analyzed the incidence of a primary diagnosis of depression among Medicare beneficiaries in the US diagnosed with wAMD during a 2-year follow-up period [16]. In this study, the diagnosis of depression was identified from the enrolment information and Medicare claims filed on behalf of beneficiaries. This study reported no significant differences in the incidence of depression between patients receiving anti-VEGF treatments and those who did not. Furthermore, a first diagnosis of depression during the follow-up period only occurred in $2.0 \%$ of the whole sample.

Finally, a recent prospective observational study conducted in Israel found a significant correlation between patients' anxiety levels experienced before the injection and pain experienced when receiving the injection [17]. In this study, $25 \%$ of the participants reported high levels of anxiety measured by a visual analogue scale (score $\geq 6$ on a scale from 0 to 10 ).

\section{Discussion}

Our review shows that the psychological impact of anti-VEGF treatments for wAMD remains a relatively new topic with limited evidence and therefore requires more research. To date, the psychological impact of receiving anti-VEGF treatments for wAMD has been addressed in studies focused on the patient experience of receiving these treatments, the impact of anti-VEGF treatments for patients' vision-related quality of life, and mental health.

The patient experience of receiving anti-VEGF has been the most addressed topic in previous studies about the psychological impact of these treatments for wAMD. In this topic, we found five articles [5-9] and one systematic review in which ten articles were reviewed [4]. Findings suggested that, in general, this treatment is well tolerated by patients, but a great portion of them were still anxious about the treatment. According to these studies, patients feel anxious especially because of previous expectations of receiving a needle in the eye, fear of losing their sight, fear of any side effects, and prior experiences of pain when receiving intravitreal injections. Furthermore, as a recent study highlighted [5], anti-VEGF treatments can have significantly changed the way patients experience this disease and cope with the fear for blindness, because now patients come across with a treatment with great potential for halting disease progression. Studies conducted prior to availability of anti-VEGF treatments considered a patients' optimism about the medical treatments and disease progression as false hope and a non-adapted behavior [25], but patients' cautious optimism about the disease progression can now be acceptable in the light of positive outcomes offered by anti-VEGF treatments and the way the wAMD prognosis is now communicated to patients [5]. However, there are some aspects of the experience of receiving anti-VEGF treatments needing more attention. In this review, we only found three studies exploring in-depth patients' experiences of receiving anti-VEGF treatments using a qualitative design $[5,7,9]$. The remaining studies that have addressed the experience of receiving anti-VEGF treatment were mainly focused on pain caused by the treatment. It is of paramount importance to generate more evidence on how patients experience these treatments, especially through qualitative research, which could yield high-quality information and is a field as yet largely unexplored [4]. Such evidence is crucial to understand patients' main sources of anxiety and what strategies patients activate to effectively cope with these treatments.

The literature we reviewed suggested that anti-VEGF treatments can have a positive impact on patients' vision-related quality of life, albeit such a relationship is dependent on 
improvements in VA [10-13]. In these studies, the concept of vision-related quality of life included not only a patient's aspects related to the use of vision in activities of everyday life but also mental health and social functioning. These findings therefore suggested a strong link between VA and patients' perceptions of quality of life, functioning, and mental health. However, further research should clarify this link as, according to a recent systematic review, not all previous studies found a strong relationship between VA and adjustment to vision loss or depression after vision loss [26].

There is strong evidence that AMD patients are likely to be depressed and anxious as a consequence of limitations imposed by vision loss $[1,2,27,28]$. Both conditions are regarded as the most frequent mental health problems among people with AMD with rates ranging from 15.7 to $44 \%$ for depressive symptoms and 9.6 to $30.1 \%$ for anxiety symptoms [2]. Depression would be an additional source of disability for these patients and can compromise the response to the treatment and medical outcomes $[1,2,29]$. Previous studies found high comorbidity of depression and AMD but most of them did not control for patients who were receiving anti-VEGF treatments $[20,29]$. It is important to consider the potential influence of anti-VEGF treatments on prevalence of depression because some studies have suggested a positive correlation between visual acuity and adjustment to vision loss [26-28], mental health [10-13], and quality of life [10-13]. In our review, we found three studies addressing depression after receiving anti-VEGF treatments for wAMD [14-17]. Two of them suggest no influence of anti-VEGF treatments on prevalence rates of depression. However, in one of these studies [15], findings should be carefully analyzed because: (a) the authors did not provide any details on how depression was measured and diagnosed during the follow-up period; and (b) the incidence rates of depression found in this study are oddly lower than those found in previous studies in which standardized and valid instruments were used to measured depression [2]. Finally, the only study that assessed the levels of anxiety associated with receiving anti-VEGF injections suggested that anti-VEGF treatment can induce clinical anxiety, and that the levels of anxiety found are within the range of prevalence of anxiety described in previous studies evaluating psychological impact of AMD [2].

Some limitations should be acknowledged in this review. First, only articles in English were included in this review, which can be considered a limitation because there are studies on the topic published in other languages. Second, the terminology used to perform the search for articles reflects not only the state of the art but also our previous experience and perhaps bias in the field. Finally, this review did not include grey literature such as academic dissertations or conference abstracts, and therefore there could be other studies addressing this topic that were not reviewed.

In conclusion, there is still very little knowledge on the psychological impact of anti-VEGF treatments for wAMD. Future studies, especially longitudinal research, should bring more evidence on this topic and clarify the positive and /or negative impact that this treatment can have for patients' mental health and quality of life. An awareness of the likely psychological impact on individual patients should allow physicians to decide holistically, which are the most appropriate treatment strategies to adopt. Such knowledge will be key to developing evidencebased clinical strategies to help patients to manage the treatment and reduce anxiety and pain levels along the treatment. An exploration of depression in patients receiving anti-VEGF treatments would also be valuable for developing new intervention strategies to prevent long-term mental health problems among these patients. Additionally, it will be helpful to prevent any patient drop-outs, which can compromise any positive outcomes brought by anti-VEGF treatments. Because ant-VEGF treatments are being used with other ophthalmologic conditions rather than wAMD, it would be relevant to study experiences of treatment, adherence to treatment, mental health, and quality of life across other conditions such as diabetic macular edema or choroidal neovascularization secondary to pathologic myopia. The specific case of macular edema deserves special attention because there is evidence of a strong link between diabetes and depression, regardless the co-existence of vision loss [30-32].

Great advances have been made over the last decade in treating a generation of patients for whom there had previously been no hope for visual gain and further research on psychological impact of anti-VEGF treatments should expand on such advances for the overall welfare and benefit of patients with retinal disease.

\section{Compliance with ethical standards}

Funding Bayer provided financial support in the form of a research grant (Reference R117779) to investigate the psychological impact of anti-VEGF treatments for wAMD. The sponsor had no role in the design or conduct of this research.

Conflict of interest The authors have the following affiliations/ involvements with organizations or entities with financial interests:

Hugo Senra is receiving an educational grant from Bayer.

Zaria Ali has no conflicts of interest.

Konstantinos Balaskas is receiving travel/educational grants from Novartis, Bayer, and Alimera.

Tariq Aslam is receiving travel/educational grants from Bayer, Novartis, Bausch and Lomb, Alimera, Oraya, and Thea.

Ethical approval All procedures performed in studies involving human participants were in accordance with the ethical standards of the institutional and/or national research committee and with the 1964 Helsinki Declaration and its later amendments or comparable ethical standards.

Open Access This article is distributed under the terms of the Creative Commons Attribution 4.0 International License (http:// creativecommons.org/licenses/by/4.0/), which permits unrestricted use, distribution, and reproduction in any medium, provided you give appropriate credit to the original author(s) and the source, provide a link to the Creative Commons license, and indicate if changes were made. 


\section{References}

1. Coleman H, Chan C, Ferris F, Chew E (2008) Age-related macular degeneration. Lancet 372:1835-1845

2. Dawson SR, Mallen CD, Gouldstone MB, Yarham R, Mansell G (2014) The prevalence of anxiety and depression in people with age-related macular degeneration: a systematic review of observational study data. BMC Ophthalmol 14:78

3. Gillies MC, Campain A, Barthelmes D, Simpson JM, Arnold JJ, Guymer RH, McAllister IL, Essex RW, Morlet N, Hunyor AP (2015) Fight retinal blindness study group (*). Long-term outcomes of treatment of neovascular age-related macular degeneration: data from an observational study. Ophthalmology 18

4. Boyle J, Vukicevic M, Koklanis K, Itsiopoulos C (2015) Experiences of patients undergoing anti-VEGF treatment for neovascular age-related macular degeneration: a systematic review. Psychol Health Med 20:296-310

5. McCloud C, Khadka J, Gilhotra JS, Pesudovs K (2014) Divergence in the lived experience of people with macular degeneration. Optom Vis Sci 91:966-974

6. Chua P, Mitrut I, Armbrecht A, Vani A, Aslam T, Dhillon B (2009) Evaluating patient discomfort, anxiety, and fear before and after ranibizumab intravitreous injection for wet age-related macular degeneration. Arch Ophthalmol 127:939-940

7. Henriksen K, Adhami S (2010) Patient experience of intravitreal injections in AMD. Int J Ophthalmic Pract 1:68-72

8. Tailor R, Beasley R, Yang Y, Narendran N (2011) Evaluation of patients' experiences at different stages of the intravitreal injection procedure-What can be improved? Clin Ophthalmol 5:1499

9. Thetford C, Hodge S, Harding S, Taylor S, Knox P (2013) Living with age-related macular degeneration treatment: patient experiences of being treated with ranibizumab (Lucentis) ${ }^{\circledR}$ intravitreal injections. Br J Vis Impair 31:89-101

10. Wang LL, Liu WJ, Liu HY, Xu X (2015) Single-site baseline and short-term outcomes of clinical characteristics and life quality evaluation of Chinese wet age-related macular degeneration patients in routine clinical practice. Chin Med J (Engl) 128:1154-1159

11. Finger RP, Wiedemann P, Blumhagen F, Pohl K, Holz FG (2013) Treatment patterns, visual acuity and quality-of-life outcomes of the WAVE study - a noninterventional study of ranibizumab treatment for neovascular age-related macular degeneration in Germany. Acta Ophthalmol 91:540-546

12. Finger RP, Guymer RH, Gillies MC, Keeffe JE (2014) The impact of anti-vascular endothelial growth factor treatment on quality of life in neovascular age-related macular degeneration. Ophthalmology 121:1246-1251

13. Inoue M, Arakawa A, Yamane S, Kadonosono K (2014) Intravitreal injection of ranibizumab using a pro re nata regimen for age-related macular degeneration and vision-related quality of life. Clin Ophthalmol 8:1711-1716

14. Casten R, Rovner BW, Leiby BE, Tasman W (2010) Depression despite anti-vascular endothelial growth factor treatment of agerelated macular degeneration. Arch Ophthalmol 128:506-508

15. Lee WJ, Cho HY, Kim DH, Yu HG, Oh J, Kim JS, Moon SW (2013) Depression of late age-related macular degeneration patients in Korea. Asia Pac J Ophthalmol 2:23-27
16. Sloan FA, Hanrahan BW (2014) The effects of technological advances on outcomes for elderly persons with exudative age-related macular degeneration. JAMA Ophthalmol 132:456-463

17. Segal O, Segal-Trivitz Y, Nemet AY, Cohen P, Geffen N, Mimouni $M(2015)$ Anxiety levels and perceived pain intensity during intravitreal injections. Acta Ophthalmol. doi:10.1111/aos.12802

18. LaHood B, Sherwood D, Suter A (2011) Comparative assessment of the effectiveness of anaesthesia for intravitreal bevacizumab injection. Clin Exp Ophthalmol 39:184-185

19. Moisseiev E, Regenbogen M, Bartfeld Y, Barak A (2012) Evaluation of pain in intravitreal bevacizumab injections. Curr Eye Res 37:813-817

20. Rifkin L, Schaal S (2012) Factors affecting patients' pain intensity during in office intravitreal injection procedure. Retina 32:696-700

21. Roth D, Scott I, Gulati N, Prenner J (2006) Patient perceptions of discomfort and changes in vision and functional status associated with intravitreal triamcinolone injection. Am J Ophthalmol 142: 492-494

22. Yau G, Jackman C, Hooper P, Sheidow T (2011) Intravitreal injection anesthesia - Comparison of different topical agents: a prospective randomized controlled trial. Am J Ophthalmol 151:333337.e2, e332

23. Mangione CM, Lee PP, Gutierrez PR, Spritzer K, Berry S, Hays RD (2001) Development of the 25-item National Eye Institute visual function questionnaire. Arch Ophthalmol 119:1050-1058

24. Yesavage JA, Brink TL, Rose TL, Lum O, Huang V, Adey MB, Leirer VO (1983) Development and validation of a geriatric depression screening scale: a preliminary report. J Psychiatr Res 17:37-49

25. Bennion AE, Shaw RL, Gibson JM (2012) What do we know about the experience of age related macular degeneration? a systematic review and meta-synthesis of qualitative research. Soc Sci Med 75: 976-985

26. Senra H, Barbosa F, Ferreira P, Vieira CR, Perrin PB, Rogers H, Rivera D, Leal I (2015) Psychologic adjustment to irreversible vision loss in adults: a systematic review. Ophthalmology 122:851861

27. Augustin A, Sahel JA, Bandello F, Dardennes R, Maurel F, Negrini C, Hieke K, Berdeaux G (2007) Anxiety and depression prevalence rates in age-related macular degeneration. Invest Ophthalmol Vis Sci 48:1498-1503

28. Eramudugolla R, Wood J, Anstey KJ (2013) Co-morbidity of depression and anxiety in common age-related eye diseases: a population-based study of 662 adults. Front Aging Neurosci 5:56

29. Casten RJ, Rovner BW (2013) Update on depression and agerelated macular degeneration. Curr Opin Ophthalmol 24:239-243

30. Kan C, Silva N, Golden SH, Rajala U, Timonen M, Stahl D, Ismail K (2013) A systematic review and meta-analysis of the association between depression and insulin resistance. Diabetes Care 36:480489

31. Winkley K, Sallis H, Kariyawasam D, Leelarathna LH, Chalder T, Edmonds ME, Stahl D, Ismail K (2012) Five-year follow-up of a cohort of people with their first diabetic foot ulcer: the persistent role of depression on mortality. Diabetologia 55:303-310

32. Kariyasam DK, Leelarathna L, Winkley K, Stahl D, Ismail K, Edmonds M (2009) Long-term follow-up of a cohort of patients with diabetes and their first foot ulcer-effect of depression on mortality. Diabetologia 52(suppl1):PS 1002-S393, Abstract 